\title{
Selected problems of gas-liquid flow through the channels filled with metal foams
}

\author{
Roman Dyga ${ }^{1}$, and Małgorzata Płaczek ${ }^{1, *}$ \\ ${ }^{1}$ Opole University of Technology, Faculty of Mechanical Engineering, Department of Process Engineering, 5 Mikolajczyka Street, \\ 45-271 Opole, Poland
}

\begin{abstract}
Open-cell metal foams are relatively unknown type of cellular material, which is increasingly being used as structural packing in the industrial equipment. The paper presents an analysis of experimental results on heat transfer and hydrodynamics of gas-liquid two-phase flow through the channels filled with metal foams. The research included the registration of temperature and pressure changes on fluid flow path. Furthermore the phase void fraction was measured and flow patterns in present in the channel were observed. It was found that in two-phase flow, both from heat transfer and hydrodynamics of flow point of view, the liquid plays the dominant role. It was also found a significant influence of flow patterns on frictional pressure loss value and phase void fraction. Whereas the flow patterns and geometry of the foam in much lesser extent influence on the intensity of heat transfer. Type of gas-liquid flow patterns primarily depends on flow conditions, including the velocity and properties of fluids. On the other hand, it wasn't stated any significant effect of geometrical parameters of foam on the type of flow and the value of phase void fraction. Among many flow patterns observed during the study, there have been identified four basic structures: plug, semi-slug, slug, and stratified.
\end{abstract}

\section{Introduction}

Various processes in technology require a maximum surface applied for heat or mass transfer. For this purpose, process apparatus often contains a variety of materials with a considerable specific surface, such as open-cell metal foams. Metal foam materials are the ones in which metal occurs in the form of thin fibers forming a cellular skeleton. Cells are the large empty areas between the fibers. The adjacent cells are interconnected by a number of "windows". The size (diameter) of a cell is usually greater by an order of magnitude from the cross-sectional size of the fibers in the skeleton. Such a design provides a very high porosity of foams, as it often exceeds $90 \%$ and it enables relatively free transport of fluids through the materials. The high thermal conductivity of metals results in the relatively high value of the effective thermal conductivity coefficient. Due to the continuous structure of the skeleton fibers, there is no thermal resistance on the contact surface between them, which normally occurs in structural packings. With the current technology, foams can be manufactured from a wide range of metal alloys. The available range of the foams includes ones from the materials, which enable their application in extremely adverse environmental conditions, such as high temperature and chemically corrosive environment.

Due to the above characteristics of metal foams, these materials are most commonly applied to play the role of elements increasing the active surface of the compact heat exchangers $[1,2]$, heat regenerators, $[3,4]$ and heat storage systems $[5,6]$. Besides, foams are applied as packings in chemical reactors [7-9]. The existing technology of catalyst deposition on the surface of the skeleton is also at a satisfactory level [10-12]. This, in turn, provides a potential to apply foams in catalytic reactors. The examples of the applications of foams in the process apparatus are given in the literature [e.g. 13,14,15].

\subsection{Gas-liquid flow through open-cell metal foams}

Two-phase gas-liquid mixture flow can be applied in industrial apparatus in which the structural packing comprises metal foams. This occurs e.g. in evaporator channels [16-18] and in column apparatus [19,20].

The results of various works contain a conclusion that the presence of foam on the boiling surface reduces the superheating temperature of this surface and shifts the boiling crisis in the direction of the greater densities of the heat flux $[21,22]$. Hu et al. [16] state that the value of the heat transfer coefficient registered during the flow of a boiling coolant through a flat channel with a metal foam packing is between 1.5-2.6 times greater in comparison to a channel that does not contain such a packing. Even a greater difference, i.e. by $2-4$ times for the advantage of the foam occurs for the case of boiling in round, tubular channels. The authors in the work [16]

Corresponding author: m.placzek@po.opole.pl 
also note that the presence of metal foam results in a considerable increase of the pressure drop of the fluid, which in turn contributes to the decrease of the efficiency of the cooling system. The assessment by $\mathrm{Hu}$ et al. [23] provides an insight that the pressure drop of a boiling mixture tend to increase along with its greater flow rate and in the conditions when the mass flow rate of the steam is greater. Similar conclusions were derived by $\mathrm{Ji}$ and $\mathrm{Xu}$ [17]. These authors registered a linear change in the pressure drop in the function of the vapor mass fraction and greater pressure drop values in a case of foams with a greater pore packing density.

A report by Wang et al. [18] contains a conclusion that the increase of the intensity of the heat transfer during boiling in an area occupied by a metal foam results from disturbances of the flow within near-wall region, more intensive mixing of the steam and the liquid as well as an increase of the effective heat transfer coefficient.

A comparison of the heat transfer in the column with various types of packing, conducted by Pangarkar et al. [19] demonstrates that metal foams can be put on the list of the category of packings in which the heat transfer occurs with a high intensity. Another advantage resulting from the application of foams in the packed columns (used in the place of other packings) is associated with the decrease of the pressure drop. According to a study by Weijiang Zhang et al. [24], pressure drop resulting from the flow accounts for only around $20 \%$ of the total pressures drop in a wire mesh packing. The relatively small pressure drop was also registered by other authors [25]. The reports based on research conducted with regard to the columns with metal foam packings generally contain a conclusion that the flow is generally much more stable in this case in comparison to flows through columns with other types of packing, e.g. in the form of a fixed bed. Due to the high porosity, it is possible to apply a greater load of the gas and liquid, whereas the volume fraction of the liquid can reach as much as the porosity of the foam, i.e. above $90 \%$ [25].

The above information is derived from the scarce sources containing reports into two-phase flow through metal foams. Due to the scarcity of such studies, the subject of gas liquid flow through metal foams can be considered as insufficiently studied and researched. The literature does not contain information regarding the flow patterns resulting from the gas-liquid flow through metal foams. The current state-of-the-art in the area faces considerable difficulties in the design, optimization and development of the apparatus containing metal foam packings. All of this formed an incentive to perform a study with regard to the identification of the phenomena that are specific with regard to the gas-liquid flow through channels with metal foam packings. A particular emphasis was placed on the determination of the impact of the flow conditions and geometrical parameters of the foams on the characteristics of the heat exchange, pressure drop and volume fraction of the phases. Another important issue taken into consideration was associated with the identification of the flow patterns during gas-liquid flow and the conditions.

\section{Scope of the experiment}

The scope of the experiment involved an experiment with regard to flow of two types of gas-liquid mixtures through horizontal channels with various types of metal foam packing. The study applied three foams made of aluminum alloys - two made of AlSi7Mg alloy and one made of Al 6101 alloy. The classification of the foams applied the density of pore packing, which according to the manufacturer's specifications was equal to 20 and 30 PPI (pore per inch) for the case of AlSi7Mg foams and 40 PPI for Al 6101 foam. All foams were characterize by similar values of the porosity $\phi$ and similar geometrical cellular skeleton, with a note that for the case of 40 PPI foam there were local deformations of the skeleton, as it is visible in Fig. 1d. The foams were distinct in terms of the cell diameter $d_{c}$ as well as pore diameter $d_{p}$ (the term pore used here applies to the window between the adjacent cells - Fig. 2c). The characteristic foam parameters are summarized in Table 1.
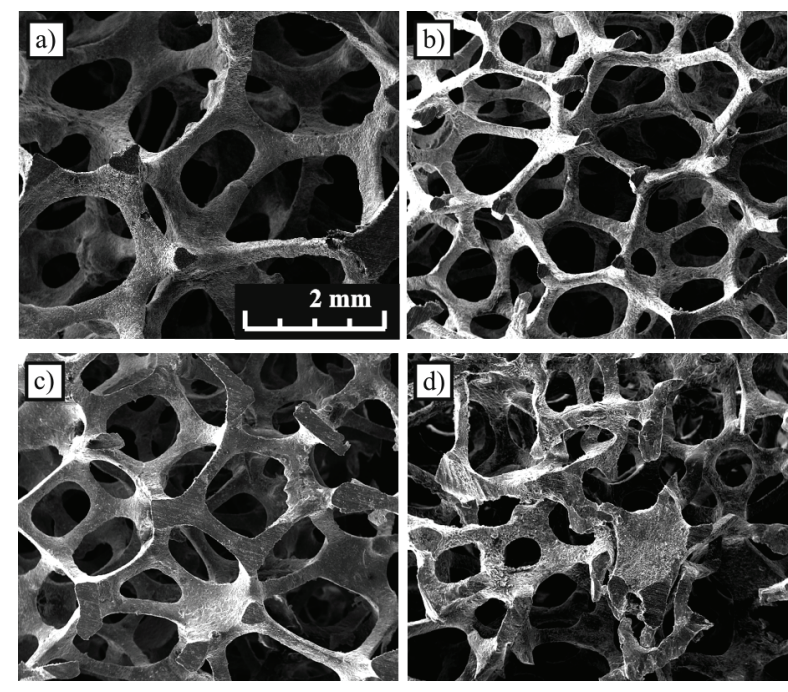

Fig. 1. Structure of the foams: a) 20 PPI, b) 30 PPI, c) 40 PPI, d) local deformations of the foam skeleton in 40 PPI foam.

Table 1. Characteristics of the foams.

\begin{tabular}{|c|c|c|c|c|}
\hline $\begin{array}{c}\text { Foam } \\
(\text { alloy })\end{array}$ & $\phi[-]$ & $\begin{array}{c}d_{c} \cdot 10^{-3} \\
{[\mathrm{~m}]}\end{array}$ & $\begin{array}{c}d_{p} \cdot 10^{-3} \\
{[\mathrm{~m}]}\end{array}$ & $\begin{array}{c}k_{s} \\
{[\mathrm{~W} /(\mathrm{m} \cdot \mathrm{K})]}\end{array}$ \\
\hline $\begin{array}{c}20 \mathrm{PPI} \\
(\mathrm{AlSi} \mathrm{Mg})\end{array}$ & 0.9336 & 3.452 & 1.094 & 150.4 \\
\hline $\begin{array}{c}30 \mathrm{PPI} \\
(\mathrm{AlSi} \mathrm{Mg})\end{array}$ & 0.9435 & 2.255 & 0.712 & 150.4 \\
\hline $\begin{array}{c}40 \mathrm{PPI} \\
(\mathrm{A} 16101)\end{array}$ & 0.9292 & 2.386 & 0.824 & 189.4 \\
\hline
\end{tabular}

The two-phase mixtures were formed by the air and water and air and Velol-9Q machine oil. From the point of view of the focus of this study, the most meaningful aspects are associated with the differences in the density, viscosity, specific heat and thermal conductivity of the components of the two-phase mixture. For oil at $20^{\circ} \mathrm{C}$, these properties are as follows: $\mu_{o l}=0.0086 \mathrm{~Pa} \cdot \mathrm{s}$, $\rho_{o l}=859.4 \quad \mathrm{~kg} / \mathrm{m}^{3}, \quad c_{o l}=1848.8 \quad \mathrm{~J} /(\mathrm{kg} \cdot \mathrm{K}), \quad k_{o l}=0.128$ $\mathrm{W} /(\mathrm{m} \cdot \mathrm{K})$. 
The study was conducted by application of an experimental installation, whose basic component was a horizontal channel with the internal diameter of $0.02 \mathrm{~m}$ completely filled with metal foam (individual channel was used for each type of the foam). In the central part of the channel, there was a measurement section $K 2$ with a length of $1.27 \mathrm{~m}$. This section was preceded by a section used for flow development, i.e. K1. Following the measurement section, there was a section comprising an outlet, $K 3$ made from plexiglas. The transparent section made it possible to conduct observations of the patterns formed during the two-phase flow. The measurement section was heated from outside along the length of $1.18 \mathrm{~m}$ by means of resistance heater wound around the channel. In the heated channel section, the metal foam is fixed to the wall of the channel by means of an epoxy-aluminum thermo-adhesive. The measurement section was insulated by mineral wool with a thickness of $0.07 \mathrm{~m}$. The diagram of the installation including the location of the measurement apparatus is presented in Fig. 2, whereas the actual view on the measurement section is found in Fig. 3.

Prior to entering the channel, the volume flow rates of the fluids forming the two-phase mixture $V_{f}$ was measured by means of various types of flowmeters with a high level of accuracy. The pressure drop $(\Delta P / \Delta L)_{2 f}$ was measured as the difference of the measured pressure in two points in the channel located at a distance of $1 \mathrm{~m}$ from each other. The measurements of the pressure drop were realized by the application of five piezoresistance sensors with the total measurement range of $0 \div 150000$ $\mathrm{Pa}$.

For the purposes of determining the air density, super pressure was measured in the channel as well.

The volume fraction of the phases $\varepsilon_{f}$ were measured by the so-called trap method. By application of the pneumatically controlled membrane valves $V P$, the entry and outlet region of the measurement section were closed at the same instant in time. Throughout such measurements, the measured volume of the liquid in relation to the volume of the measurement section corresponded to the volume fraction of the liquid in the gas-liquid mixture. The flow patterns were identified on the basis of visual observations. The presence of foam in the channel resulted in a considerable deterioration of the visibility, hence, the attempt to visualize the results by use of the photographic and motion picture techniques did not offer satisfactory results.

The scope of the research into heat transport involved the measurement of liquid temperature $t_{f}$ and the channel wall $t_{b}$ by means of type $\mathrm{K}$ thermocouples with the diameter of $1 \mathrm{~mm}$. The temperatures of the liquid and the wall were measured at four points along the channel length (sections I, II, III and IV in Fig. 2). Each of the sections comprised eight thermocouples. The tips of five thermocouples were located inside the channel at various distances from its axis (in the vertical plane). In turn the tips of three thermocouples were located inside the channel wall at a distance of $0.5 \mathrm{~mm}$ from its internal surface.

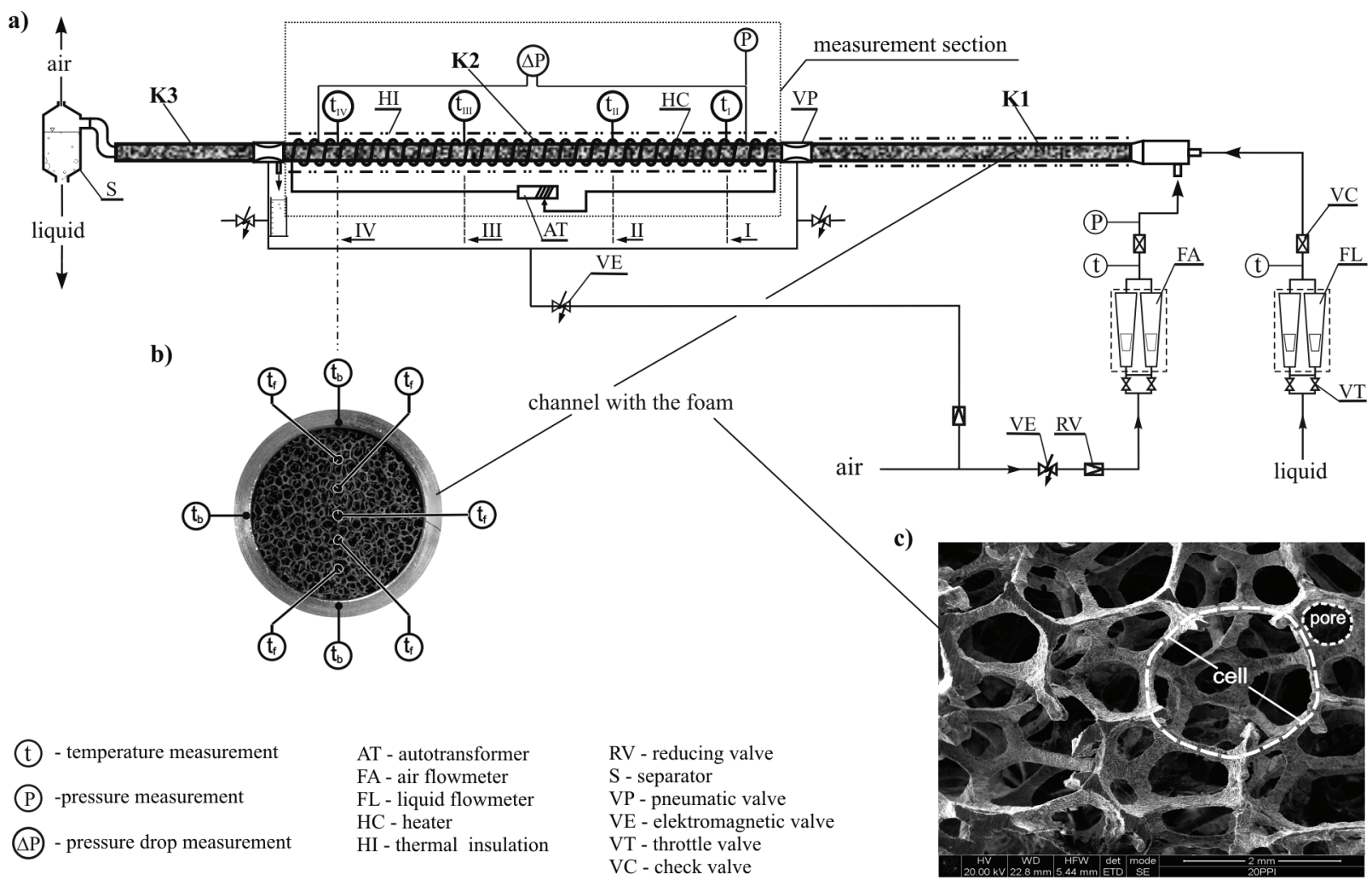

Fig. 2. Experimental setup, a) diagram of measurement section, b) location of thermocouples in the channel cross-section, c) details of cell and pore diameters. 


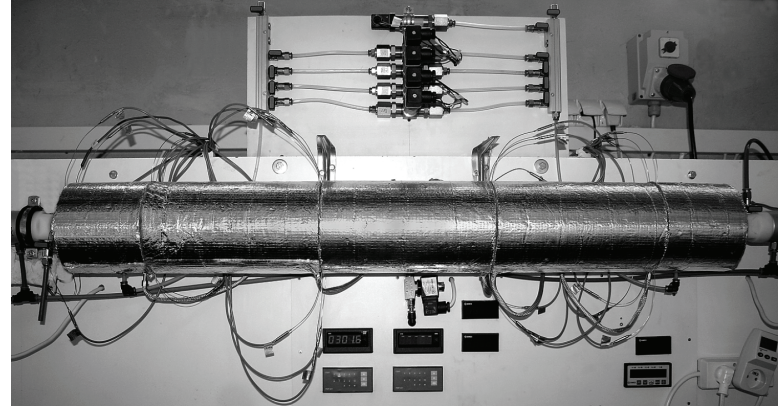

Fig. 3. Measurement section of the experimental setup.

The measurements were conducted within a considerable range of the fluid velocities (Table 2) so that laminar and turbulent flow conditions were recorded. The superficial phase velocity $w_{f, o}$ was applied to characterize the flow conditions. This notion denotes the mean velocity of the fluid flow through an empty measurement channel (i.e. when the impact of the presence of the other fluid and foam is disregarded),

$$
w_{f}=\frac{4 V_{f}}{\pi d^{2}}, \quad f \equiv a, o l, w
$$

where: $V_{f}-$ volume flow rate of fluid $\left[\mathrm{m}^{3} / \mathrm{s}\right]$, $d$ - internal channel diameter [m].

Table 2. Conditions of conducted experiment.

\begin{tabular}{|c|c|c|c|}
\hline fluid, $f$ & $w_{f}[\mathrm{~m} / \mathrm{s}]$ & $\zeta_{f}[-]$ & $t_{f}\left[{ }^{\circ} \mathrm{C}\right]$ \\
\hline air, $a$ & $0.028-2.39$ & $0.313-0.998$ & $21-95$ \\
\hline water, $w$ & $0.006-0.061$ & $0.002-0.988$ & $24-88$ \\
\hline oil, $o l$ & $0.006-0.061$ & $0.002-0.988$ & $19-93$ \\
\hline
\end{tabular}

$\zeta_{f}$ - inlet volume fraction of phases, i.e. ratio of the volume flow rate of a given phase in relations to the volume flow rate of gas-liquid mixture at the entry region to the channel.

\section{Analysis of the results}

\subsection{Flow patterns}

A number of various flow patterns were registered during the experiment. It was stated that the types of the forming patterns depend on the mutual relations of the flow rates and their properties. In the same flow conditions, i.e. for the same velocity and volume fraction of the phases, nearly identical flow patterns were observed for all foams (for given gas and liquid mixtures). Hence, the study did not demonstrate the impact of the geometrical parameters of foams on the gas-liquid flow patterns.

Four basic types of flow were identified on the basis of the formed patterns - stratified, plug, semislug and slug flows. In addition, other, transient forms of these flows were observed, such as stratified-semislug and plug-slug flows.

In the stratified flow, both phases flow in the form of continuous layers (Fig. 4a), with a thickness that is relative to the volume flow rate of the fluids at the inlet to the channel. Depending on the phase velocities, the interfacial surface can demonstrate wavy characteristics to a various degree. In extreme cases, the accumulation of the liquid can occupy the entire cross-section of the channel, forming semislug flow pattern. This type of flow was observed for the case when the superficial velocity of the liquid was in excess of $0.03 \mathrm{~m} / \mathrm{s}$, and the air velocity was greater than $1 \mathrm{~m} / \mathrm{s}$.

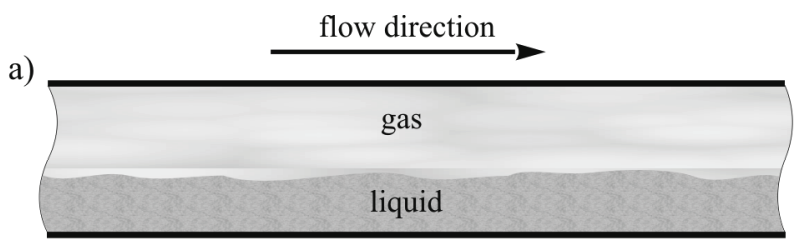

b)

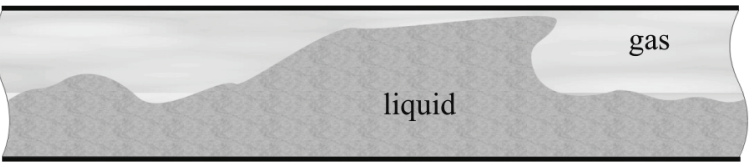

c)

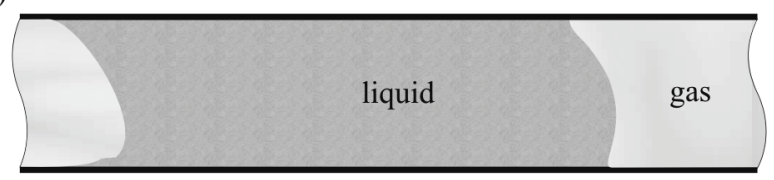

d)

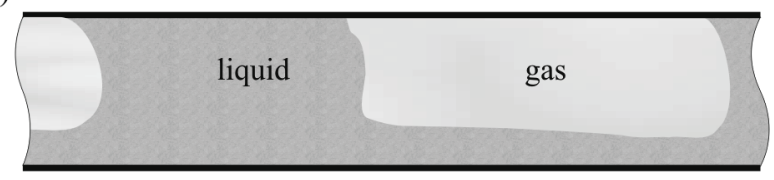

e)

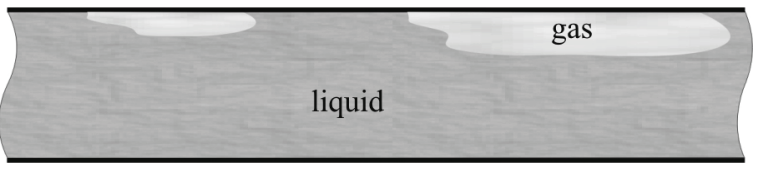

Fig. 4. Gas-liquid flow patterns in a channel with aluminum foam packing: a) stratified, b) semislug, c) slug, d) e) plug flow.

Very similar conditions can lead to the formation of the slug flow. Periodically, every few seconds, large liquid portions are observed in the channel, as they flow across the entire cross-section of the channel with a considerable velocity. Semislug and slug flows occur particularly frequently during the pumping of the air-oil mixture. For the case of a relatively large flow rate of oil, stratified flow was not observed at all, although it was the dominant type of flow for the air-water mixture. Stratified flow was only observed in the tests applying oil only for the case when only a small section of the channel was occupied by this liquid.

For the case of flows comprising large volume fraction of liquids and concurrently small air fraction, we have to do with the plug flow in the channel, as presented in Fig. 4d and 4e. In such a case, air occupies the top section of the channel in the form of extended bubbles, the so-called plugs. The dimensions of the plugs increase along with the increase in the flow rate of the air. Plug flow was registered for the case when the 
superficial air velocity did not exceed $0.2 \mathrm{~m} / \mathrm{s}$, and the ratio of the liquid to gas velocities was at least equal to 0.5 .

On the basis of the sole values of the phase velocities, or else their volume fractions in the mixture, there is no possibility to unambiguously indicate the type of flow that is formed in the given flow conditions. The formation of the patterns is determined by a number of factors, including the physical properties of the fluids. During an attempt to identify a manner in which the particular flow patterns are formed in the channels with metal foam packing, it was established that this is possible to achieve it in a reference system proposed by Baker $\left(g_{c} / g_{g}\right) \gamma_{B} \Psi_{B}-g_{g} / \gamma_{B}$. In such a system, $g_{c}$ and $g_{g}$ denote the density of the mass flow rate of liquid and gas, respectively, and dimensionless parameters $\gamma_{B}$ and $\psi_{B}$ account for the relations between the physical properties of the gas and liquid to air and water,

$$
\begin{gathered}
\gamma_{B}=\left[\left(\frac{\rho_{g}}{\rho_{a}}\right)\left(\frac{\rho_{l}}{\rho_{w}}\right)\right]^{0.5}, \\
\psi_{B}=\left(\frac{\sigma_{w}}{\sigma_{l}}\right)\left\{\left[\left(\frac{\mu_{l}}{\mu_{w}}\right)\left(\frac{\rho_{w}}{\rho_{l}}\right)\right]^{2}\right\}^{1 / 3},
\end{gathered}
$$

where: $\rho, \mu, \sigma$ denote the density $\left[\mathrm{kg} / \mathrm{m}^{3}\right]$, viscosity $[\mathrm{Pa} \cdot \mathrm{s}]$ and surface tension $[\mathrm{N} / \mathrm{m}]$, respectively, while the subscripts refer to: $l$ - liquid, $g-$ gas, $a-$ air, $w$ - water. As we can see in Fig. 5, the point denoting the type of the flow pattern form the compact regions (in a given reference system) determining the flow conditions in which the particular flow patterns can occur.

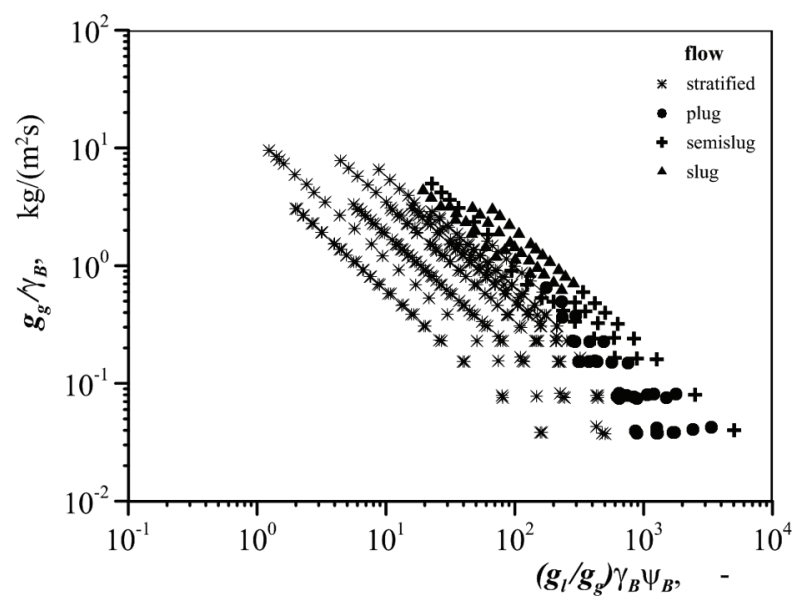

Fig. 5. Conditions of gas-liquid flow patterns occurrence during flow through metal foams.

\subsection{Volume fractions of phases}

The results of the measured volume fraction of the phases demonstrate that the volume fraction of gas $\varepsilon_{a}$ increases along with the increase of the velocity of this phase. Concurrently, an increase of the liquid velocity contributes to decrease of the gas volume fraction. This observation is illustrated in Fig. 6, on the basis of an example involving air-water flow. The increase of the velocities of the phases results from the increase of the fluxes in the entry region of the channel; hence, the increased flow rate of the liquid in the channel leads to the reduction of the free space available for the gas (and the reverse).

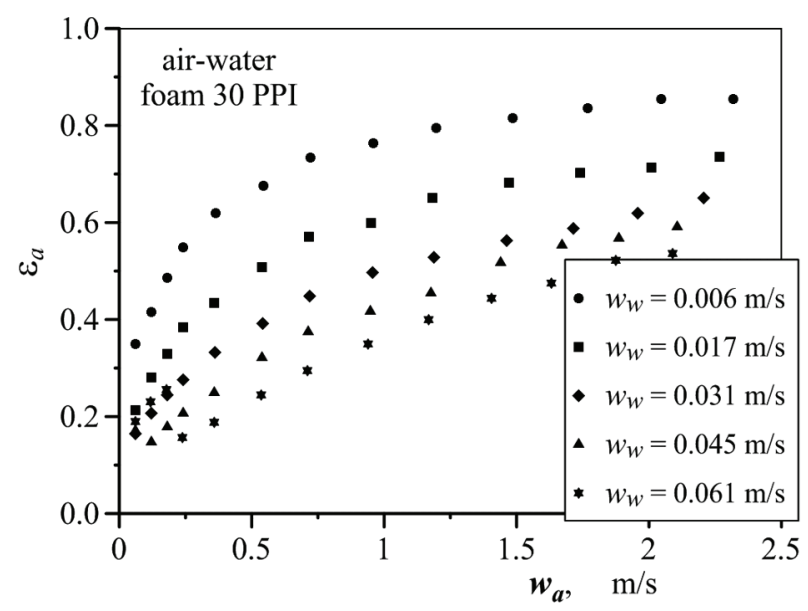

Fig. 6. Impact of phase velocities on the volume fraction of air in the mixture containing water - flow through 30 PPI foam.

For all three foams, in the same flow conditions (for a given gas-liquid mixture), the experiment offered very similar results with regard to the volume fraction of the phases (Fig. 7), which demonstrates the lack of the impact of the geometrical structure of the foam on the registered volume fractions.

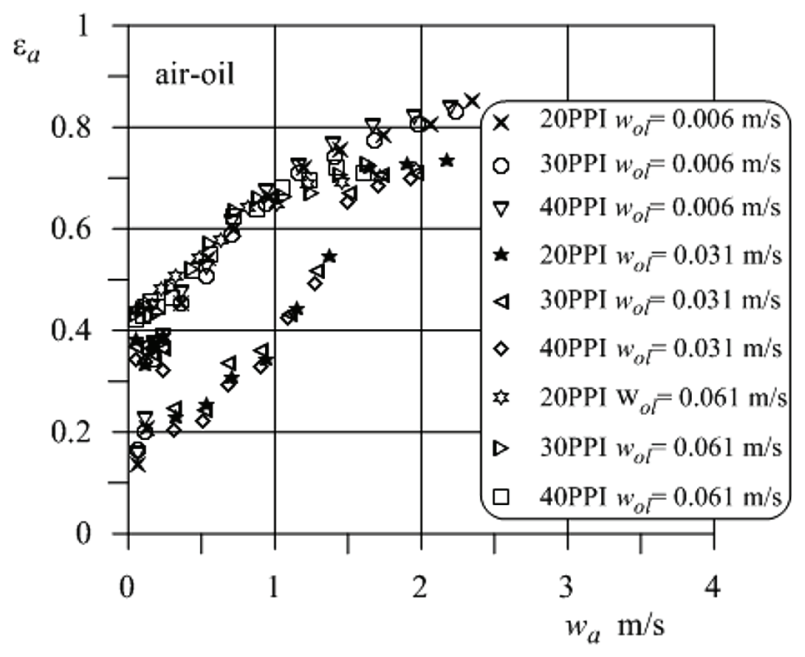

Fig. 7. Comparison of air volume fraction in the air-oil mixture during flow through 20,30 and 40 PPI foams.

Foam forms the reason for the considerable interfacial slip, which is evidenced by the considerable differences between the inlet volume fraction in the entry region to the channel $\zeta_{f}$ and volume fraction of the phases in the region occupied by the foam $\varepsilon_{f}$. The air passes relatively freely through the space occupied by the foam due to its small viscosity. The foam skeleton tends to stop the more viscous liquid (in particular this regards oil). As a consequence, the volume fraction of the air in the space occupied by the foam could be over 
four times smaller from its inlet volume fraction (Fig. 8). In addition, as demonstrated in a work by [26], the volume fraction of the gas in the mixture pumped through the foams is smaller than for the case of the identical flow through empty channels.

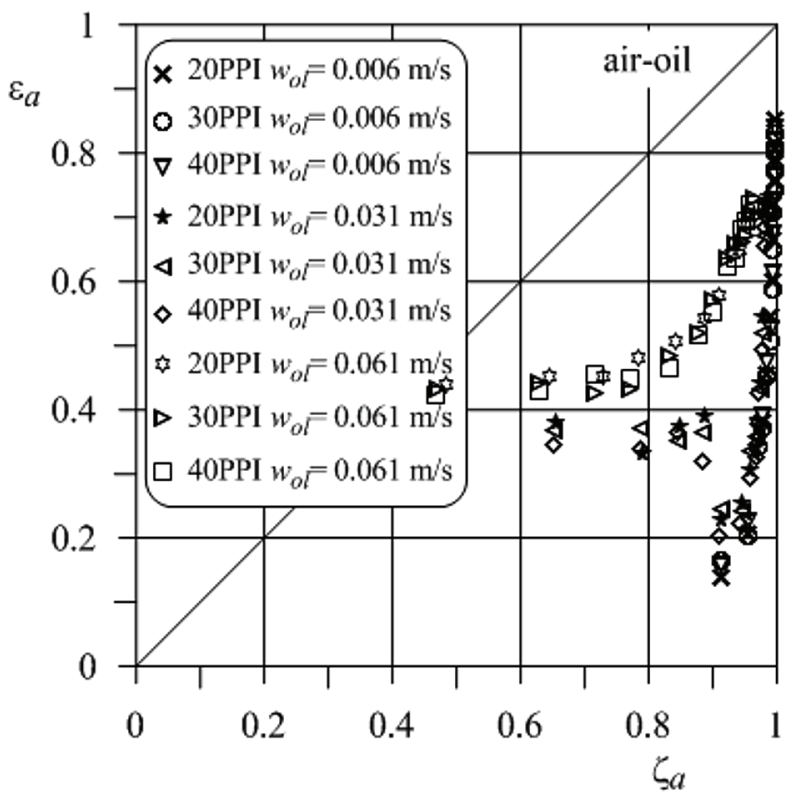

Fig. 8. Comparison of the measured volume fraction of air with its inlet volume fraction.

Despite the monotonous increase along with the increase of the velocities of the phases, the volume fraction of the phases tends to change abruptly when a change of the flow pattern occurs. This phenomenon is particularly evidenced for the change of the plug flow into the stratified one (Fig. 9); nevertheless, similar phenomena could also be registered for other flow patterns.

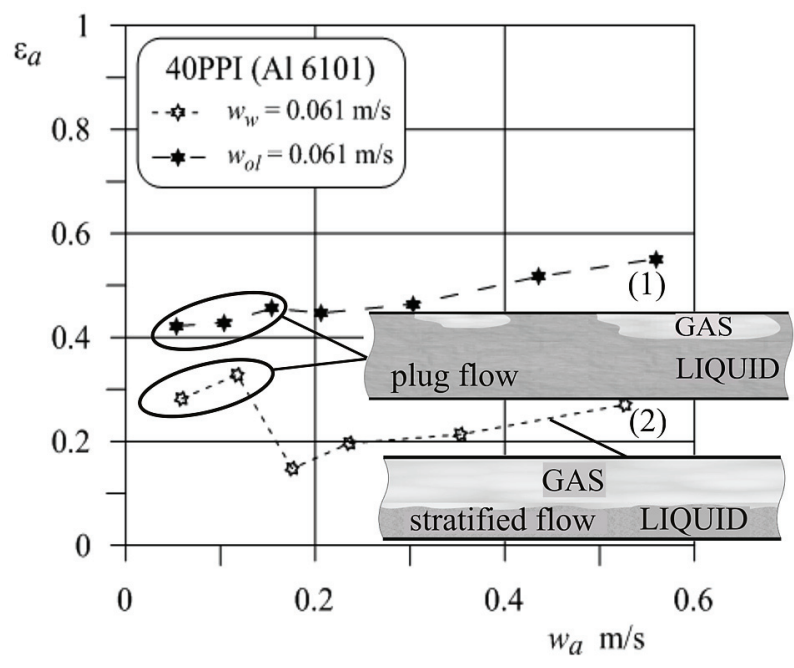

Fig. 9. Course of the changes in the air volume fraction in the conditions of flow patterns change during air-water and air-oil flow.

\subsection{Pressure drop}

Pressure drop during two-phase flow $(\Delta P / \Delta L)_{2 f}$ increases significantly along with the increase of the gas and liquid velocities. For a small flow rate of the air, pressure drop tends to vary almost linearly and only when this velocity exceeds $1 \mathrm{~m} / \mathrm{s}$, the variations in the pressure drop take on exponential values. The deviations from the monotonous increase of the pressure drop occur (similarly as in case of the volume fraction) for the case when a change of the flow patterns occurs. An example of this could be associated with the apparently unjustified decrease of the value $(\Delta P / \Delta L)_{2 f}$ despite an increase of the air velocity in the conditions of the transformation of plug flow into the stratified one (Fig. 10). The decrease of the pressure drop, which occurs in this case results from the decrease of the channel and foam surface that is wetted by water. In the plug flow, the liquid is in contact with a considerably greater surface than for the case of the stratified flow. Because water is a more viscous phase than air, it decides on the values of the pressure drop during two-phase flow to a greater degree than air does.

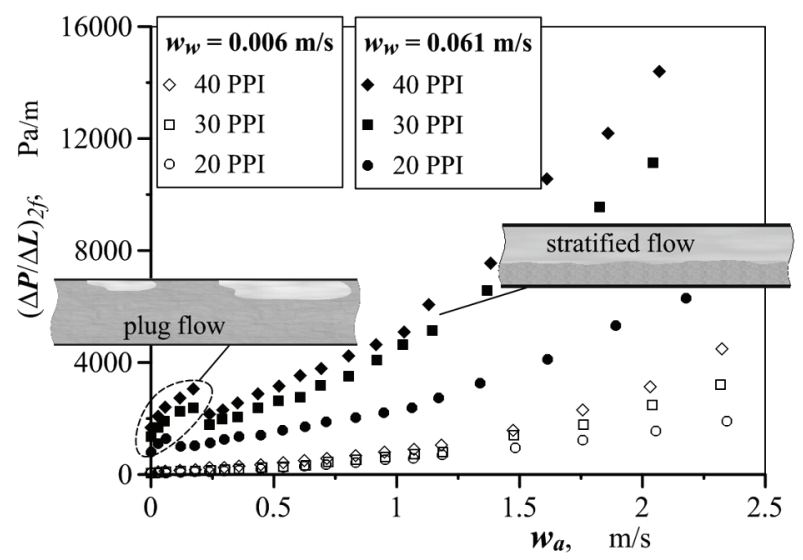

Fig. 10. Air-water pressure drop (accounting for the reduction effect) during flow through 20,30 and 40 PPI foams.

This type of peculiarities are more frequently noted for the case of the air-oil mixture flow. This is due to the greater diversity of the flow patterns during this type of flow (as air-water flows mainly take the form of a stratified flow), which in turn leads to a more irregular course of pressure drop. The values of the pressure drop during air-oil mixture flow are also considerably greater than in the case of the air-water flow. This is due to the greater oil viscosity.

During flow of two-phase mixture through channels with various types of foams, different values of pressure drop were registered (in the same conditions). This means that the value of the pressure drop is affected by the geometrical parameters of foam. The smallest values of pressure drop were registered during flow through 20 PPI foam, whereas the greatest - for the flow through the 40 PPI foam (Fig. 10). The 20 PPI foam is characterized by the biggest cells diameter, which means that the flow occurs more freely than for the case of foams with the smaller cells. Although the cell diameter in 40 PPI foams is slightly larger than for the case of 30 PPI foam, the value of the pressure drop during flow through the first 
of them is considerably greater than for the case of the 30 PPI foams. The reason is likely to be associated with the specific geometrical structure of the 40 PPI foam skeleton. The large nodes at the contact points of the fibers in this foam, which are discernible in Fig. 1c, along with the numerous deformations of the skeleton (Fig. 1d) undoubtedly lead to an increase of the pressure drop.

\subsection{Heat transfer}

The measurement of temperature offered the determination of heat transfer coefficient value $\alpha_{2 f}$ from the heated channel walls to the two-phase fluid,

$$
\alpha_{2 f}=\frac{q_{2 f}}{t_{b}-t_{f}},
$$

where $t_{b}$ and $t_{f}$ denote the mean temperatures of the wall and the fluid calculated on the basis of data from all temperature measurement points located along the heated channel section (i.e. 32 in total).

The heat flux $q_{2 f}$ is given by the total of the densities of the heat fluxes absorbed by the liquids $\left(q_{w}\right.$ or $\left.q_{o l}\right)$ and that of the air $q_{a}$. This value was derived on the basis of the heat balances of the particular fluids

$$
q_{f}=m_{f}\left(i_{f}^{k}-i_{f}^{p}\right), \quad f \equiv a, o l, w
$$

The increase of the enthalpy of fluids $\left(i_{f}^{k}-i_{f}^{p}\right)$ was calculated on the basis of the temperature of the twophase mixture at the entry and outlet regions of the measurement section. For the case of the air-water flow, the heat balance accounts for the latent heat of the evaporated water as well.

For all instances of flow that were investigated, the heat transfer coefficient increases along with the increase of the velocity of each of the phases. In addition, as we can observe in Fig. 11, the value of the heat transfer coefficient during two-phase flow is greater from the case when single-phase liquid flow is taken into account $\left(w_{a}=0\right)$.

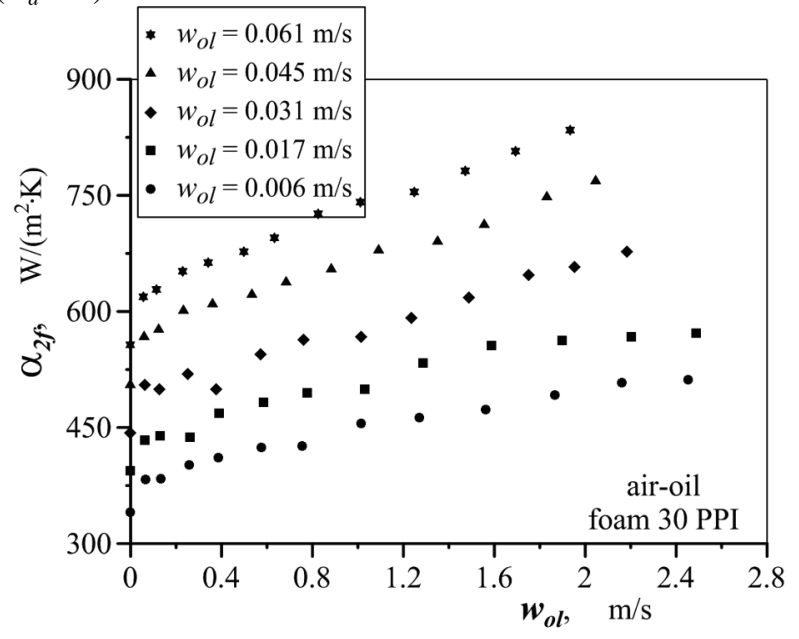

Fig. 11. Heat transfer coefficient during water-oil flow through a channel with 30 PPI foam packing
In contrast to the measurements with regard to the pressure drop and volume fraction, for the case of the studies involving heat transfer, the studies did not confirm the significant impact of the flow patterns on the intensity of heat transfer.

The value of heat transfer coefficient depends on the type of foam - its geometrical parameters and heat conductivity.

The greatest values of $\alpha_{2 f}$ were recorded for the flow through 20 PPI foam, whereas the lowest- for the flow through 30 PPI foam (Fig. 12). In the consideration of the specific surface of the foam, which in combination with the internal area of channel form the surface of the heat exchange, the heat transfer coefficient should assume the greatest values for the case of the 30 PPI foam. This is due to the greater specific surface in this foam compared to 20 PPI foam.

The reverse result obtained in the study was explained in another paper [27]. In this paper, it was demonstrated that in the channels with metal foam packing, the heat exchange occurs primarily as a result of the heat transfer from the channel walls directly into the fluid, i.e. excluding the role of the foam. Foam itself participates in the heat transfer mainly through the conduction. During the flow through 40 PPI foam, the heat transfer coefficient assumes intermediate values, i.e. ones between the results gained for 20 and 30 PPI foams. In terms of its geometry, 40 PPI foam is similar to 30 PPI (i.e. they both have similar cell and pore size); however, it has a considerably greater value of the thermal conductivity $k_{s}$ equal to $189 \mathrm{~W} /(\mathrm{m} \cdot \mathrm{K})$, compared to $k_{s}=150.4 \mathrm{~W} /(\mathrm{m} \cdot \mathrm{K})$ for $20 \mathrm{PPI}$ and $30 \mathrm{PPI}$ foams. A part of the heat is conducted through the foam skeleton and it is transferred to the fluid through it. Hence, the considerable thermal conductivity of 40 PPI foam contributes to the greater intensity of heat transfer compared to the case of flow through 30 PPI foam with a similar geometry.

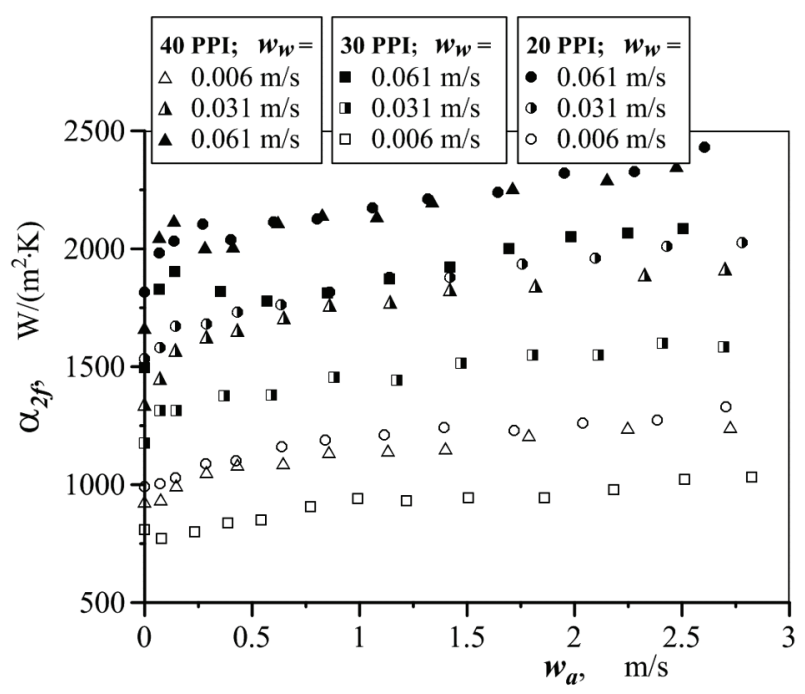

Fig. 12. Comparison of heat transfer coefficient for 20, 30 and 40 PPI foams in the conditions of air-water flow 


\section{Conclusions}

The conducted experiments and analysis of the results demonstrate that the hydrodynamic and thermal phenomena related to the two-phase gas-liquid flow through channels packed with open-cell metal foams are determined principally by the flow conditions, including velocities of the fluids and their physical properties. The structure of foams affects the quantities characterizing the flow to a smaller degree - in some cases the impact of the foam structure can be even disregarded. For instance, the presence of foam forms a reason for the occurrence of interfacial slip which is the same for various foams (within the range of the performed experiment); hence, the structure of the foam does not affect the value of the volume fraction of the phases. The foam does not lead to the dispersion of the phases either, which is familiar from the flow through porous packings. During the flow through the foams, standard patterns are formed, such that are typical for flow through empty channels.

Four principal types of flow patterns were identified: plug, semislug, slug and stratified flow. The type of the flow patterns is determined only by the type of the flow conditions (i.e. velocities, properties and volume fraction of the phases). In addition, the formed patterns are independent of the geometrical parameters of the foams through which the flow occurs.

The parameters of the foams have a moderate impact on the characteristics of heat transfer and considerable effect on pressure drop during two-phase flow. From heat transfer point of view, the most important aspect is associated with thermal conductivity of foam material. The pressure drop during fluid flow through channel with metal foam packing is relative to the porosity of the foam, cell size and layout of the cell skeleton (apart from the well- recognized effect of the velocity and fluid properties). The pressure drop during two-phase flow and volume fraction of the phases change monotonously along with the changing velocities of the phases. Considerable deviations from this regularity can be observed in the conditions of the changes in the flow patterns.

\section{References}

1. E.J. Cookson, D.E. Floyd, A.J. Shih, Int. J. Mechanical Sci. 48 (2006)

2. B. Ozmat, B. Leyda, B. Benson, Mater. Manuf. Processes 19, 5 (2004)

3. Z.Q. Chen, P. Cheng, T.S. Zhao, Int. Commun. Heat Mass 27, 3 (2000)

4. K. Wang, Y.L. Ju, X.S. Lu, A.Z. Gu, Cryogenics, 47 (2007)

5. Y. Tian, C.Y. Zhao, Int. J. Heat Mass Transf. 58 (2013)

6. P.H. Vadwala, Thermal Energy Storage in Copper Foams filled with Paraffin Wax (Master of Applied Science, Mechanical \& Industrial Engineering University of Toronto, 2011)
7. J. Lévêque, D. Rouzineau, M. Prévost, M. Meyer, Chem. Eng. Sci. 64 (2009)

8. R. Tschentscher, M. Schubert, A. Bieberle, T.A. Nijhuis, J. van der Schaaf, U. Hampel, J.C. Schouten, Chem. Eng. Sci. 66 (2011)

9. G. Incera Garrido, F.C. Patcas, S. Lang, B. Kraushaar-Czarnetzki CheM. EGg. Sci., 63 (2008)

10. M. Mitov, R. Rashkov, N. Atanassov, A. Zielonka, J Mater. Sci. 42 (2007)

11. A. Sirijaruphan, J.G. Goodwin jr., R.W. Rice, D. Wei, K.R. Butcher, G.W. Roberts, J.J. Spivey, Appl. Catal. A: Gen. 281 (2005)

12. H. Yu, H. Chen, M. Pan, Y. Tang, K. Zeng, F. Peng, H. Wang, Appl. Catal. A: Gen. 327 (2007)

13. J. Banhart, Progress Mater. Sci. 46 (2001)

14. X.-H. Han, Q. Wang, Y.-G. Park, C. T'joen, A. Sommers, A. Jacobi, Heat Transf. Eng. 33, 12 (2012)

15. C.Y. Zhao, Int. J. Heat Mass Transf. 55 (2012)

16. H. Hu, Y. Zhu, H. Peng, G. Ding, S. Sun, Appl. Therm. Eng. 62 (2014)

17. X. Ji, J. Xu, Heat Mass Transf. 48 (2012)

18. P. Wang, D.Y. Liu, C. Xu, Appl. Energy 102 (2013)

19. K. Pangarkar, T.J. Schildhauer, J.R. van Ommen, J. Nijenhuis, J.A. Moulijn, F. Kapteijn, Chem. Eng. Sci. 65 (2010)

20. S7] C.P. Stemmet, M. Meeuwse, J. van der Schaaf, B.F.M. Kuster, J.C. Schouten, Chem. Eng. Sci. 62 (2007)

21. L. Tadrist, M. Miscevic, O. Rahli, F. Topin, Exp. Thermal Fluid Sci. 28 (2004)

22. Z.G. Xu, Z.G. Qu, C.Y. Zhao, W.Q. Tao, Int. J. Heat Mass Transf. 54 (2011)

23. H. Hu, Y. Zhu, G. Ding, S. Sun, Int. J. Refrigeration 36 (2013)

24. W. Zhang, B. Zhang, Z. Shi, Procedia Eng. 18 (2011)

25. S6] C.P. Stemmet, J.N. Jongmans, J. van der Schaaf, B.F.M. Kuster, J.C. Schouten, Chem. Eng. Sci. 60 (2005)

26. M. Płaczek, R. Dyga, Chem. Eng. Equipment 4 (2013)

27. R. Dyga, L. Troniewski, Arch. Thermodyn. 36, 1 (2015) 